\title{
Relationship between ulnar collateral ligament thickness and joint hypermobility in young adults
}

\author{
Peter R. Reuter*, Arie J. van Duijn, Kaylee R. Fichthorn, Lacy Mroz
}

Department of Rehabilitation Sciences, Marieb College of Health \& Human Services, Florida Gulf Coast University, Fort Myers, Florida, USA

\section{Article Info}

\section{Article Notes}

Received: June 09, 2021

Accepted: August 06, 2021

\section{*Correspondence:}

${ }^{*}$ Dr. Peter R. Reuter, Department of Rehabilitation Sciences, Marieb College of Health \& Human Services, Florida Gulf Coast University, Fort Myers, Florida, USA; Email: preuter@fgcu.edu

${ }^{\ominus} 2021$ Reuter PR. This article is distributed under the terms of the Creative Commons Attribution 4.0 International License.

\section{Keywords}

Joint hypermobility

Beighton score

Elbow joint

Ulnar collateral ligament

Anterior bundle thickness

Elbow joint width

Valgus stress

\section{Abstract}

Joint hypermobility is largely understood as a dysfunction of collagen fibers within connective tissues, allowing for a range of motion markedly increased over validated normal values. The ulnar collateral ligament (UCL) of the elbow provides stability to the elbow during valgus stress and, thus, has great significance in overhead throwing sports due to its susceptibility to injury. Our cross-sectional study explored the relationship between the length and width of the anterior bundle of the ulnar collateral ligament of the elbow and joint hypermobility. Two hundred and eighty-four undergraduate students completed a Beighton score assessment. Ultrasound images of the participants' UCL were obtained in both arms at rest and under gravity induced valgus force. Sixty-one participants reported hypermobility in at least one elbow joint, and hypermobility in one elbow joint was correlated with hypermobility in the other. There were moderate correlations between $\mathrm{UCL}$ thickness and joint gapping between left and right elbow joints. However, there was no significant difference in UCL anterior bundle thickness or medial elbow joint gapping at rest or under valgus stress between those with and without hypermobile elbow joints. The thickness of the anterior bundle of the UCL and humeroulnar joint gap at rest moderately correlates to corresponding thickness and width under valgus stress. There is no significant difference in UCL anterior bundle thickness or joint width in people with elbow hypermobility and those without.

\section{Introduction}

Joint hypermobility is typically defined as the ability for a joint to move beyond the normal range of motion ${ }^{1}$. The Beighton score as a method for determining joint hypermobility was described by Beighton, Solomon and Soskolne ${ }^{2}$ in 1973. Based on the 2017 recommendations of the International Consortium on the EhlersDanlos syndromes (EDS), the diagnosis of generalized joint hypermobility $(\mathrm{GJH})$ requires a Beighton score of $\geq 5 / 9$ for pubertal men and women up to the age of 50 , a score of $\geq 6 / 9$ in pre-pubertal children and adolescents, and a score of $\geq 4 / 9$ for people older than 50 years of age ${ }^{3}$.

While many people with joint hypermobility remain free from clinical symptoms, such as musculoskeletal injuries or pain ${ }^{4,5}$, others suffer from joint hypermobility-related disorders ranging from hypermobile Ehlers-Danlos Syndrome (hEDS) to Hypermobility Spectrum Disorders ${ }^{6,7}$. Joint hypermobility in patients with Heritable Disorders of Connective Tissue (HDCTs) has long been understood as a dysfunction of the connective tissue on the cellular level ${ }^{8}$. Several defects in connective tissue proteins, including tenascin $\mathrm{X}$ in addition to type I, III, and V collagen, have been described ${ }^{9}$. Naturally, collagen dysfunction goes beyond the musculoskeletal system and 
may cause cardiovascular disorders, such as an increased risk of aortic root dilation ${ }^{8}$, as well as neurological, gastrointestinal, dermatological, and immunological signs and symptoms ${ }^{10}$. Consequently, it has been hypothesized that asymptomatic forms of joint hypermobility, such as $\mathrm{GJH}$, are less severe variants of the same dysfunction. Specifically, joint hypermobility has been addressed as a decrease in the diameter of collagen fibrils due to heritable gene mutation ${ }^{8}$.

Previous research was limited in providing insight into how the characteristics of the structures surrounding specific joints, such as the width of ligaments and tendons, may be affected by joint hypermobility ${ }^{8}$. One study, for example, identified a correlation between a narrow width of the anterior cruciate ligament of the knee and an increase in anterior knee laxity in recreationally active females ${ }^{11}$. In another study, GJH was positively correlated with radiographically determined laxity of the trapeziometacarpal joint ${ }^{12}$.

The ulnar collateral ligament (UCL) provides stability to the elbow during valgus stress. The ligament is composed of three separate bundles (anterior, posterior, and transverse) with different attachment sites and fiber directions ${ }^{13}$. The anterior bundle is the longest and strongest of these bundles; it runs in an oblique fashion from humerus to ulna. The posterior bundle is a shorter bundle that runs vertically from humerus to ulna. The transverse bundle is thought to provide little to no contribution to valgus support due to its horizontal orientation that does not cross the humeroulnar joint line. Due to their differing attachments, the posterior and anterior bands work together in a reciprocal fashion; the anterior bundle is most taut in extension while the posterior bundle is most taut in flexion ${ }^{14}$. The anterior bundle is considered as the greatest contributor of medial elbow joint stability against valgus stress ${ }^{15}$.

Due to its susceptibility to injury, the UCL has great significance in overhead throwing sports ${ }^{16,17}$. During the late cocking and early acceleration phases of overhead throwing, the elbow undergoes a valgus movement that places significant stress on the medial compartment and the UCL. Consequently, the UCL has been frequently researched in athletic populations such as baseball pitchers ${ }^{18,19}$.

Previously published research, however, has neglected to examine the role of generalized hypermobility on these structures, or the laxity of the humeroulnar joint specifically. The purpose of this study was to examine the relationship between elbow joint hypermobility, UCL thickness, and joint gapping measured using sonography to build upon the body of knowledge of the contributing factors and characteristics of joint hypermobility, specifically in a population of college students ages 18-25.

As of writing, no study was identified that examined the characteristics of the UCL in a joint with an innately increased range of motion. The anatomic differences observed as a result of the chronic repetitive trauma of overhand throwing sports, which stress the ligament past its intrinsic range of motion, may be significantly different from those differences observed in an inherently hypermobile joint. This distinction is precisely what the current study aimed to elucidate.

\section{Methods}

We performed a cross-sectional, quantitative correlational study. Participants consisted of undergraduate students enrolled in Human Anatomy \& Physiology with lab I classes at a regional university in Florida. Data collection corresponded with curricular education on the general structure and function of joints and range of motion during Fall semester 2018 and Spring semester 2019. Prior to each data collection round, members of the research team delivered a group presentation to participants informing them on the purpose of the study and instructing them on how to assess range of motion using goniometers.

A power analysis was completed in order to determine an appropriate sample size for achieving statistical significance $(p=.80$; effect size $=.30)$. In order to achieve this power, we needed to recruit 68 students between the ages of eighteen and twenty-five years and without any pathological hypermobility conditions. This sample size is representative of the estimated $14 \%$ of college students with benign joint hypermobility and based off of an estimated student population of 14,957. Due to the size of Human Anatomy \& Physiology with lab I classes, estimated participation was well above the power analysis requirement.

Participants were made aware of the voluntary and anonymous nature of the study, and their right to withdraw from the study at any time without consequence. All participants received and signed an Institutional Review Board (IRB) approved informed consent form. Participants then completed the self-response survey and Beighton score assessment.

\section{Self-Response Survey and Beighton Score}

The anonymous self-response survey was described previously and used successfully for the same purpose ${ }^{5}$. Participants worked in pairs guided by trained members of the research team to complete a Beighton score assessment following the criteria in Table 1 using goniometers as necessary. The scoring system involves nine joints and a tested joint is either hypermobile (score $=1$ ) or shows normal mobility (score $=0$ ); therefore, the total score (i.e., the Beighton score) can range from 0 to 9 . Because of the age of the participants, a Beighton score of 5 or higher was used in this study to diagnose GJH. 
Table 1: The Beighton criteria for joint hypermobility

Passive dorsiflexion of the little fingers beyond $90^{\circ}$ (one point for each hand) - two points

Passive apposition of the thumbs to the flexor aspects of the forearm (one point for each thumb) - two points

Hyperextension of the elbows beyond $10^{\circ}$ (one point for each elbow) - two points

Hyperextension of the knee beyond $10^{\circ}$ (one point for each knee) - two points

Forward flexion of the trunk with knees fully extended so that the palms of the hands rest flat on the floor - one point

\section{Ultrasound Imaging}

Ultrasound imaging to determine ligament thickness and to perform joint gapping measurements has been found to demonstrate high reliability and inter-rater reliability for determining absolute joint gap width, making it an appropriate method of evaluating the structural characteristics of the elbow joint ${ }^{20}$. Studies comparing ultrasound imaging to magnetic resonance imaging (MRI) and computed tomography noted the sensitivity of ultrasound for diagnosing UCL injuries ranged from 86$100 \%$, suggesting optimal visibility of the UCL ligament ${ }^{21}$.

Trained members of the research team completed ultrasound imaging studies of bilateral humeroulnar joints for each participant. Lumify portable ultrasound with $\mathrm{L}$ 12-4 broadband linear array transducer technology was used to capture an image of each participant's right and left elbow in open-packed position. Each image captured the trochlear origin of the UCL and the humeroulnar joint space in the visual field. For the second ultrasound image the participants' elbows were placed under valgus stress, i.e., the humeroulnar joint was placed in 60 degrees of flexion, the shoulder abducted off the edge of the table, and relaxed into external rotation. This position allows sufficient gravity driven valgus force on the humeroulnar joint ${ }^{22}$. The humeroulnar joint was captured in this position with the same field of view criteria as in open-packed position. Open-packed and valgus stressed images were captured for both elbows for a total of four images per participant. OsiriX DICOM imaging software was used to independently measure the midsubstance thickness of the anterior bundle of the UCL, as well as humeroulnar joint gapping in each of the four images obtained.

\section{Data analysis}

Data collection yielded both categorical and parametric data. Due to the voluntary nature of the survey, sample sizes varied for each analysis. All data analysis was performed using SPSS (Statistical Package for Social Sciences), version 24, IBS Corporation, 2016, Armonk, NY.

Descriptive data analysis was performed to describe characteristics of participants in the sample. Pearson product-moment correlation coefficients were calculated to determine 1) the relationship between UCL thickness and joint gapping at rest and under valgus force, and 2) the relationship between UCL thickness and joint gapping between right and left elbows. A paired t-test was used to determine whether the application of valgus load had a significant effect on UCL thickness or on joint width.

For analysis involving GJH, we subdivided participants into two groups, one containing participants with GJH with a Beighton score of $\geq 5$ and one with participants without GJH with a Beighton score of $<5$. Multivariate analysis of variance (MANOVA) was used to analyze differences in anterior bundle thickness at rest and under valgus force, and humeroulnar joint gapping at rest in those with and without elbow hypermobility and GJH. Chi-square tests were used to determine 1) the relationship between right and left elbow hypermobility and 2) the relationship between elbow hypermobility and GJH.

\section{Results}

Of 330 respondents, 44 were excluded due to age requirements or failure to report their age; two respondents were excluded due to reporting connective tissue disorders, leaving a 284-participant sample for analysis (Table 2).

The average thickness for the anterior bundle of the UCL and the average width of the humeroulnar joint for open-packed position and under valgus stress are reported in Table 3. For each elbow, UCL thickness at rest was moderately correlated with UCL thickness under valgus force (right: $r=0.530, p<0.001$; left: $r=0.491, p<0.001$ ). Humeroulnar joint gap at rest and under valgus stress showed a weaker but statistically significant relationship in both elbows (right: $r=0.391, p<0.001$; left: 0.243 , $p$ $<0.001$ ). We found moderate correlations between UCL thickness and joint gapping between left and right elbows (e.g., left to right UCL thickness under valgus: $r=0.414, p<$ 0.001 ; left to right joint width: $\mathrm{r}=0.315, \mathrm{p}<0.001$ ). Gravity induced valgus load led to small changes in joint widths of both elbows but this change was only significant for the left elbow $(\Delta$ right $=0.005, \mathrm{p}=0.149 ; \Delta$ left $=0.015, \mathrm{p}=<0.001)$.

Table 2: Study population profile

Biological sex: $80.6 \%$ female students, $19.4 \%$ male students

Age: $19.0 \pm 1.27$ years (mean \pm standard deviation; range: 18 - 25 years; median age: 19 years

Race/ethnicity: 68.0\% Caucasian/White, 11.6\% Hispanic, 8.5\% African-American/Black, 11.9\% more than one ethnicity/race or ethnicity/ race other than listed

Handedness: $86.0 \%$ right-handed, $12.0 \%$ left-handed, $2.0 \%$ ambidextrous 
Table 3: Average thickness of anterior bundle of UCL and width of humeroulnar joint in $\mathrm{mm} \pm$ standard deviation (SD) for open-packed position and under valgus stress

\begin{tabular}{|c|c|c|c|}
\hline \multicolumn{4}{|c|}{ Thickness anterior bundle of UCL $[\mathrm{mm} \pm \mathrm{SD}]$} \\
\hline \multicolumn{2}{|c|}{ Right elbow joint } & \multicolumn{2}{|c|}{ Left elbow joint } \\
\hline open-packed position & $3.39 \pm 0.73$ & open-packed position & $3.41 \pm 0.69$ \\
\hline under valgus stress & $3.38 \pm 0.73$ & under valgus stress & $3.47 \pm 0.74$ \\
\hline \multicolumn{4}{|c|}{ Width of humeroulnar joint (humeroulnar joint gap) [mm \pm SD] } \\
\hline \multicolumn{2}{|c|}{ Right elbow joint } & \multicolumn{2}{|c|}{ Left elbow joint } \\
\hline open-packed position & $2.26 \pm 0.65$ & open-packed position & $2.27 \pm 0.66$ \\
\hline under valgus stress & $2.31 \pm 0.69$ & under valgus stress & $2.42 \pm 0.67$ \\
\hline
\end{tabular}

Sixty-one participants $(21.5 \%)$ reported hypermobility in at least one elbow joint; hypermobility in one elbow joint was strongly correlated with hypermobility in the other elbow joint $\left(x^{2}=196.190, p<0.001\right)$. One in seven participants $(14.4 \% ; \mathrm{n}=41)$ reported $\mathrm{GJH}$ based on a Beighton score $\geq 5$.

There was no significant difference in joint characteristics at rest or under valgus stress between participants with hypermobile elbow joints and those without (right: Wilks' Lambda $=0.987, F_{4,224}, \mathrm{p}=0.551$; left: Wilks' Lambda $=0.990, F_{4,242}, \mathrm{p}=0.654$ ). There was, however, a correlation between left elbow joint gapping under valgus stress and $\mathrm{GJH}$ at the 0.05 level $\left(F_{1}=0.4889\right.$, $\mathrm{p}=0.028$ ), although this was a weak relationship (Etasquared $=0.023$ ). We found no other relationship between the joint characteristics of either elbow and GJH.

Nonetheless, there were significant differences (right $t_{84.7}=10.930, \mathrm{p}<.001$, left $t_{75.2}=10.18, \mathrm{p}<.001$ ) between Beighton scores for participants with hypermobile elbow joints (right: $\mathrm{M}=4.33, \mathrm{SD}=1.91$; left: $\mathrm{M}=4.39, \mathrm{SD}=2.10$ ) and those with normal elbow joints (right: $\mathrm{M}=1.40, \mathrm{SD}=1.61$; left: $M=1.41, S D=1.54) ; \quad$ We found similarly significant differences between classification of GJH and the presence of elbow joint hypermobility (right: $\mathrm{x}_{1}{ }_{1}=65.738, \mathrm{p}<.001$; left $\mathrm{x}_{1}^{2}=62.265, \mathrm{p}<.001$ ).

There were no significant differences in UCL thickness and joint gapping at rest or under valgus stress among participants with hypermobile elbow joints and those without (right: $F_{4,224}=0.987, \mathrm{p}=0.551$; left: $F_{4,242}=0.990$, $\mathrm{p}=0.654$ ). Conversely, there was a significant difference in left elbow joint gapping under valgus stress among participants with GJH and those without $(F=0.4889, \mathrm{p}=$ $0.028, \Delta$ right $=0.005, \mathrm{p}=0.149 ; \Delta \mathrm{left}=0.015, \mathrm{p}=<0.001$ ).

\section{Discussion}

This study adds to the body of knowledge pertaining to the characteristics of and contributing factors to joint hypermobility. The thickness of the anterior UCL bundle at rest was related with UCL thickness under valgus force and a similar relationship was found for humeroulnar joint width under the same conditions. Additionally, participants with hypermobility in one elbow were likely to report hypermobility in both elbows, somewhat in keeping with the pervasive consequences of cellular collagen fibril disruption. However, there were no relationships between elbow hypermobility and elbow joint characteristics or their distortion under valgus stress. GJH was not strongly related to elbow joint characteristics at rest or under valgus stress. We further discuss our sample characteristics and the implications of our results.

The mean widths of the humeroulnar joint gap (right: $2.26 \mathrm{~mm}$; left: $2.27 \mathrm{~mm}$ ) of our participants confirm previously published results ${ }^{23}$. Published studies reported the thickness of the anterior bundle of the UCL to be between 4 and $7.6 \mathrm{~mm}^{13,14,15,21,23}$. Our measurements of thickness for both elbow joints (right: $3.39 \mathrm{~mm}$; left: 3.41 $\mathrm{mm}$ ) come in lower to this range. But, with one exception ${ }^{21}$, previously published studies regarding UCL anterior bundle thickness were comprised primarily of cadaver studies, were consequently limited in sample size, and results may differ from in vivo studies such as our own.

Our study did not find a significant difference in anterior bundle thickness in participants with hypermobile elbow joints compared with participants with non-hypermobile elbow joints. As of writing, no study that examined joint characteristics and morphological changes of the UCL in subjects with asymptomatic hypermobility was identified. Studies examining valgus instability in the elbows of professional baseball players have noted an increase in thickness of the anterior bundle of the UCL ${ }^{10,18,24}$. However, as previously mentioned, the authors of these studies suggest the increased thickness may be a result of calcification or fluid buildup in the ligament due to repetitive trauma. The UCL characteristics of asymptomatic participants with underlying hypermobility may therefore be different from the study populations of these studies who undergo repetitive stress to the medial elbow compartment daily.

We found no significant difference between the joint width in hypermobile elbows versus normal. Previous studies deliver varying results of changes in joint width under valgus stress. In a study of asymptomatic professional baseball pitchers $(n=70)$, humeroulnar joint width under valgus force was greater in participants who injured their elbow in the following season vs. those who did not (6.5 
mm vs. $5.3 \mathrm{~mm})^{10}$. A larger study $(\mathrm{n}=122)$ of high school baseball pitchers by Tajika et al. ${ }^{24}$, did not find a significant difference in humeroulnar joint space with or without valgus stress and regardless of whether participants had a history of elbow symptoms (for example, pain or instability) or not. To our knowledge, our study is the first in vivo study to examine humeroulnar joint characteristics in hypermobile vs. non-hypermobile elbows of a nonathletic population.

Our results show differences between the joint width of right and left elbows when comparing GJH and non$\mathrm{GJH}$ groups. There was a difference in joint width for left elbows, but not for right elbows. A number of studies have reported differences between dominant and non-dominant arms $s^{6,18,25,26}$. However, the populations of these studies were comprised of overhead throwing athletes whose dominant and non-dominant elbows are placed under significantly different amounts of repetitive stress.

The differing relationships between elbows we found in our study therefore require further explanation. The role of the flexor tendons in providing assistance to the UCL against valgus stress may be an important consideration. Studies have identified significant overlap between the anterior bundle of the UCL and flexor digitorum superficialis (FDS) tendon insertion (45.6\%; 95\% CI: 38.1-53.6), and demonstrated that muscle activation of the finger flexors reduced humeroulnar joint gap under valgus stress ${ }^{25,27}$. A study by Kong et al. ${ }^{28}$ found variability in self- perceived percentages of maximum voluntary grip strength based on hand dominance; rates of contraction in the dominant hand were routinely underestimated, while rates of contraction in the non-dominant hand were routinely over-estimated. These findings may indicate a more nuanced role of FDS in providing valgus stability. Namely, since the majority of our sample was right handed, and we found the change in joint space under valgus load change was only significant for the left elbow, this theory may also allow for the possibility that participants in our study were unknowingly contracting their finger flexor muscles at different unknown rates between elbows.

\section{Study limitations}

There are two primary limitations to our study: 1) sampling method and 2) reliance on self-reported hypermobility data. Limiting the sample to an undergraduate university course limits our results to college-aged adults, i.e., the study population was not completely generalized to community populations. More importantly, Anatomy and Physiology courses are prerequisites to our institution's nursing program and, consequently, $80.6 \%$ of our participants were female.

Second, relying on self-reported measurement of joint angles, even with training in goniometer use and participants working in groups of two-four students under supervision of the research team, may lead to reporting errors. Participants may have unconsciously over-reported or under-reported hypermobile joints. However, there is no indication that participants with different Beighton scores may have misreported to varying degrees.

\section{Conclusions}

Our study examined the relationship between joint hypermobility, ulnar collateral ligament anterior bundle thickness, and humeroulnar joint gapping using diagnostic ultrasound in college students ages 18-25. The thickness of the anterior bundle of the UCL and humeroulnar joint gap at rest moderately correlates to corresponding thickness and width under valgus stress. Hypermobility in one elbow strongly predicts hypermobility in the other. There is no statistically significant difference in UCL anterior bundle thickness or joint width in people with elbow hypermobility and those without.

\section{Abbreviations}

EDS - Ehlers-Danlos syndrome

FDS - Flexor digitorum superficialis

GJH- Generalized joint hypermobility

HDCTs - Heritable Disorders of Connective Tissue

hEDS - hypermobile Ehlers-Danlos Syndrome

SD - Standard deviation

UCL - Ulnar collateral ligament

\section{Conflict of Interest}

The authors declare that there is no conflict of interest. The authors did not receive funding for this study and have full control of all primary data.

\section{Ethical Research Statement}

The research protocol was approved by the Institutional Review Board (IRB) at Florida Gulf Coast University (FGCU) prior to data collection (IRB Protocol 2014-64). All faculty and students involved in data collection completed training in ethical data collection through the Collaborative Institutional Training Initiative (CITI).

\section{References}

1. Collinge R, Jane V. Hypermobility, injury rate and rehabilitation in a professional football squad - A preliminary study. Phys Ther Sport. 2009; 10(3): 91-96.

2. Beighton P, Solomon L, Soskolne CL. Articular mobility in an African population. Ann Rheum Dis. 1973; 32(5): 413-418.

3. Malfait F, Francomano C, Byers P, et al. The 2017 international classification of the Ehlers-Danlos syndromes. Am J Med Genet C Semin Med Genet. 2017; 175(1): 8-26.

4. Reuter PR, Fichthorn KR. Prevalence of generalized joint hypermobility, musculoskeletal injuries, and chronic musculoskeletal pain among American university students. PeerJ. 2019; 7: e7625. 
5. Reuter PR. Joint hypermobility and musculoskeletal injuries in a university-aged population. Phys Ther Sport. 2021; 49: 123-128. https://doi.org/10.1016/j.ptsp.2021.02.009

6. Russek LN, Stott P, Simmonds J. Recognizing and effectively managing hypermobility-related conditions. Phys Ther. 2019; 99: 1189-1200.

7. Tuna F. Prevalence of joint hypermobility, hypermobility spectrum disorder and hypermobile Ehlers-Danlos syndrome in a university population: an observational study. Eur Res J. 2020; 6(2): 120-129. doi: 10.18621/eurj.466831

8. Wolf JM, Cameron JL, Owens BD. Impact of joint laxity and hypermobility on the musculoskeletal system. J AAOS. 2011; 19(8): 463-471.

9. Syx D, De Wandele I, Rombaut L, et al. Hypermobility, the EhlersDanlos syndromes and chronic pain. Clin Exp Rheumatol. 2017; 35: 116-122.

10. Shanley E, Smith M, Mayer BK, et al. Using stress ultrasonography to understand the risk of UCL injury among professional baseball pitchers based on ligament morphology and dynamic abnormalities. Orthop J Sports Med. 2018; 6(8): 2325967118788847.

11. Wang H, Shultz SJ, Schmitz RJ. Association of anterior cruciate ligament width with anterior knee laxity. J Athl Train. 2016; 51(6): 460-465.

12. Wolf JM, Schreier S, Tomsic S, et al. Radiographic laxity of the trapeziometacarpal joint is correlated with generalized joint hypermobility. J Hand Surg. 2011; 36(7): 1165-1169.

13. Lynch KP. Ultrasound imaging to evaluate acute adaptations of the medial elbow joint complex in college baseball pitchers. ProQuest Dissertations Publishing. 2018.

14. Yoshida M, Goto $\mathrm{H}$, Takenaga T, et al. Anterior and posterior bands of the anterior bundle in the elbow ulnar collateral ligament: ultrasound anatomy. J Shoulder Elbow Surg. 2017; 26(10): 1803-1809.

15. Dugas IR, Ostrander RV, Cain EL, et al. Anatomy of the anterior bundle of the ulnar collateral ligament J Shoulder Elbow Surg. 2007; 16: 657660. doi: 10.1016/j.jse.2006.11.009

16. Bruce JR, Andrews JR. Ulnar collateral ligament injuries in the throwing athlete. J Am Acad Orthop Surg. 2014; 22: 315-325. doi: 10.5435/JAAOS-22-05-315
17. Labott JR, Aibinder WR, Dines JS, et al. Understanding the medial ulnar collateral ligament of the elbow: Review of native ligament anatomy and function. World J Orthop. 2018; 9(6): 78-84.

18. Tajika T, Yamamoto A, Oya N, et al. The morphologic change of the ulnar collateral ligament of elbow in high school baseball pitchers, with and without symptoms, by sonography. J Shoulder Elbow Surg. 2016; 25(8): 1223-1228.

19. Atanda A, Buckley PS, Hammoud S, et al. Early anatomic changes of the ulnar collateral ligament identified by stress ultrasound of the elbow in young professional baseball pitchers. Am J Sport Med. 2015; 43(12): 2943-2949.

20. Nazarian LN, McShane JM, Ciccotti MG, et al. Dynamic US of the anterior band of the ulnar collateral ligament of the elbow in asymptomatic major league baseball pitchers. Radiology. 2003; 227(1): 149-154.

21. Slane L, Slane J, Scheys L. The measurement of medial knee gap width using ultrasound. Arch Orthop Trauma Surg. 2017; 137(8): 1121-1128.

22. Wood N, Konin JG, Nofsinger C. Diagnosis of an ulnar collateral ligament tear using musculoskeletal ultrasound in a collegiate baseball pitcher: A case report. N Am J Sports Phys Ther. 2010; 5(4): 227-233.

23. Smith B. The use of ultrasound imaging in assessing UCL width for college baseball pitchers. ProQuest Dissertations Publishing. 2016.

24. Ciccotti MC, Ciccotti MG, Cohen SB, et al. Stress ultrasound evaluation of medial elbow instability in a cadaveric model. Am J Sports Med. 2014; 42(10): 2463-2469.

25. Frangiamore SJ, Moatshe G, Kruckeberg BM, et al. Qualitative and quantitative analyses of the dynamic and static stabilizers of the medial elbow: an anatomic study. Am J Sports Med. 2018; 46(3): 687-694.

26. Iurassich S, Rocco D, Aurilia A. Type III Ehlers-Danlos syndrome: correlations among clinical signs, ultrasound, and histologic findings in a study of 35 cases. Int J Dermatol. 2001; 40(3): 175-178.

27. Peng $Q$ S Schafer $D$, Hsu J, et al. Mechanical actions of individual muscles at the human elbow joint and their roles in protecting/unloading the ulnar collateral ligament. Conf Proc IEEE Eng Med Biol Soc. 2004; 2004: 4704-4705.

28. Kong YK, Lee JH, Shin JM, et al. Evaluation of subjective perceived rating for grip strength depending on handedness for various target force levels. Work. 2019; 62(1): 21-26. 